\title{
RESEARCH ARTICLE \\ Parameter Optimization for a Novel Inerter-based Dynamic Vibration Absorber with Negative Stiffness
}

\author{
Jing $\mathrm{Li}^{1} \cdot$ Xueqing $\mathrm{Gu}^{1}$ · Shaotao $\mathrm{Zhu}^{1,2}$. Changlong $\mathrm{Yu}^{1} \cdot$ Xiaodong Yang ${ }^{3}$
}

Received: 3 December 2021 / Accepted: 12 February 2022 / Published online: 1 March 2022

(c) The Author(s) 2022

\begin{abstract}
A novel dynamic vibration absorber(DVA) model with negative stiffness and inerter-mass is presented and analytically studied in this paper. The research shows there are still two fixed points independent of the absorber damping in the amplitude frequency curve of the primary system when the system contains negative stiffness and inerter-mass. The optimum frequency ratio is obtained based on the fixed-point theory. In order to ensure the stability of the system, it is found that inappropriate inerter coefficient will cause the system instable when screening optimal negative stiffness ratio. Accordingly, the best working range of inerter is determined and optimal negative stiffness ratio and approximate optimal damping ratio are also obtained. At last the control performance of the presented DVA is compared with three existing typical DVAs. The comparison results in harmonic and random excitation show that the presented DVA could not only reduce the peak value of the amplitude-frequency curve of the primary system significantly, but also broaden the efficient frequency range of vibration mitigation.
\end{abstract}

Keywords Dynamic vibration absorber · Negative stiffness · Inerter-mass · Fixedpoint theory $\cdot$ Parameters optimization

\section{Abbreviations \\ DVA dynamic vibration absorber. \\ SDOF single degree-of-freedom.}

Jing Li

leejing@bjut.edu.cn

1 Interdisciplinary Research Institute, Faculty of Science, Beijing University of Technology, Beijing 100124, China

2 Faculty of Information Technology, Beijing University of Technology, Beijing 100124, China

3 Faculty of Materials and Manufacturing, Beijing University of Technology, Beijing 100124, China 


\section{Introduction}

Vibration control plays a critical role in industrial productions and has become one of the most important research topics in the field of nonlinear dynamics. Many types of vibration control techniques and efficient devices have been developed over the years. Dynamic vibration absorber (DVA), as one of the common devices for vibration control, has been widely used in engineering practice such as transportation, earthquake and civil engineering due to it's simple structure and low cost.

A dynamic vibration absorber is a device that reduces the vibration of a primary vibration system by absorbing the vibration energy of the primary system through control structures attached to the primary system. In recent decades, various structural forms and working methods of the DVA have been proposed. The first DVA without damping was invented by Frahm [1] in 1909. In 1928, Ormondroyd and Den Hartog [2,3] found that a DVA with damping element could suppress the amplitude of the primary system in a broader frequency range, which had been recognized as the typical Voigt type DVA. Den Hartog and Ormondroyd also proposed the optimization principle of the damped DVA in terms of minimizing the maximum amplitude response of the primary system. This optimum design method of the dynamic vibration absorber is called the fixed-points theory, which was well documented in the textbook by Den Hartog [3]. In 2001, Ren [4] presented a DVA where the damping element was not connected to the primary system, but to the earth or the base structure. The result indicated it could present better control performance than Voigt type DVA under the same parameters condition. Thereafter, Asami et al. [5-7] derived the exact series solutions for the optimum frequency and damping ratios of the DVA attached to damped linear systems.

The general definition of stiffness is the load that an elastic element bears when it produces a unit deformation. If the deformation increases with the increase of load, the stiffness is positive. If the load increases and the deformation decreases, the stiffness is negative. Devices made up of a number of basic components exhibit negative stiffness characteristics under certain conditions, such as inverted pendulum, magnetic device, pressure bar device, etc. Negative stiffness element has been widely used in system vibration reduction in recent years because of its advantages of large bearing capacity, small deformation and good controllability, and the parallel use of positive stiffness element can effectively solve the contradiction between low stiffness and high static load shape variables. Thus, it is necessary and meaningful to draw into the negative stiffness to the vibration control system. In 2013, Acar et al. [8] analyzed and experimentally studied an adaptive passive DVA with a negative stiffness mechanism and found that it can suppress the amplitude of the system by appropriately adjusting the parameters. Yang et al. [9] studied nonlinear vibration isolation system with negative stiffness mechanism by means of average method. The results show that adding negative 
stiffness can greatly enlarge the vibration isolation frequency band and achieve effective vibration isolation effect. Shen et al. [10,11] applied negative stiffness elements to a variety of vibration absorbers, proving that vibration absorbers with reasonable negative stiffness elements have significant control performance. Zhou et al. [12] proposed two configurations of dynamic vibration absorber in conjunction with negative stiffness and optimized them using $H_{\infty}$ criterion and stability maximization criterion.

With the development of practical engineering research, components with amplifying function such as inerter are applied to the vibration absorption system to obtain better performance. Inerter is a new type of structural control element which is related to the acceleration of two endpoints and is widely used in vibration control field. Wang et al. [13, 14] designed a variety of DVAs with inerter and derived their corresponding optimal parameter design formulas. Hu et al. [15, 16] investigated the influence of inerter on natural frequencies of vibration systems, and the efficiency of inerter in reducing the largest natural frequencies is verified. An improved inerter-based DVA was proposed by Gioacchino et al. [17], which combined the diamond bracket with inerter. Brzeski et al. [18] investigated a special type of inerter equipped with a continuously variable transmission and gear-ratio control system, and compared control performance with changes of mass and damping coefficient. Willian et al. [19] discussed the advantages and disadvantages of the inerter in the vibration isolation system, and proposed a simple method to improve the high frequency performance of the system. De Domenico et al. [20] proposed a vibration control system that combines a traditional foundation isolation scheme with an inertial foundation device. When inertiators are mounted in series with spring and damper elements, a lower mass and more efficient alternative to conventional tuned mass damper where device inertia plays a role in tune mass damper is obtained. Based on the sensitivity analysis, the effect of detuning is discussed.These studies show that the introduction of inerter has a potential advantage in improving the performance of the DVA. Kim et al. [21] proposed and analyzed the optimal design of friction multi-tuned mass dampers, studied the use of statistical linearization to replace the original nonlinear system and the equivalent linear system, and found a primary structure of optimal design to minimize the root mean square displacement. Sun et al. [22] studied a vibration isolation system of n-layer scissor structure, analyzed and designed nonlinear stiffness, friction and damping characteristics, and it is easy to achieve better vibration isolation performance and loading capacity by designing structural parameters. Bian et al. [23] designed a novel tunable nonlinear tuned mass damper using a bionic X-type structure. Compared with traditional absorbers, $\mathrm{X}$ absorbers can significantly improve the robustness of system parameters, expand anti-resonance and expand vibration suppression bandwidth. These studies showed that the introduction of inerter has a potential advantage in improving the performance of the DVA.

As described above, inerter can change the inertia characteristics of the system without changing the physical mass of the structure, grounding stiffness can adjust the stiffness characteristics of the system. Both devices can change 
the natural frequency of the system, and improve the performance of the vibration absorber. The control performance of the dynamic vibration absorber can be improved by using the grounded negative stiffness and inertial vessel simultaneously, but most of the current researches only introduce inertial vessel or the grounded negative stiffnes into the dynamic vibration absorber.

In this paper the effect of the negative stiffness and inerter on the amplitude of the primary system is studied by introducing inerter and the negative stiffness which is connected the DVA with the earth. Section 2 presents the model and optimizes the system parameters based on the fixed-point theory. Section 3 compares the present DVA with other typical DVAs under harmonic and random excitations, the results verify the DVA in this paper has more significant control performance. The conclusion is drawn finally in Sect. 4.

\section{The Model of DVA and Parameters Optimization}

\subsection{Dynamic Model of DVA}

Figure 1 shows the dynamic vibration absorber model proposed in this paper, where $m_{1}, m_{2}, k_{1}$ and $k_{2}$ are the masses, linear stiffness coefficients of the primary system and the DVA respectively. $c$ is the damping coefficient of the absorber, $b$ is the inertance and $k$ is the negative stiffness coefficient. $x_{1}$ and $x_{2}$ are the displacements of the primary system and the DVA respectively. $F$ and $\omega$ are the amplitude and frequency of the force excitation.

According to Newton's second law, the motion equation of the system with negative stiffness can be established as

$$
\begin{aligned}
& m_{1} \ddot{x}_{1}+\left(k_{1}+k_{2}\right) x_{1}-k_{2} x_{2}+b \ddot{x}_{1}-b \ddot{x}_{2}=F \cos (\omega t) \\
& m_{2} \ddot{x}_{2}+\left(k+k_{2}\right) x_{2}-k_{2} x_{1}+c \dot{x}_{2}-b \ddot{x}_{1}+b \ddot{x}_{2}=0
\end{aligned}
$$

Using the following parametric transformation

Fig. 1 The model of DVA with negative stiffness and inertermass

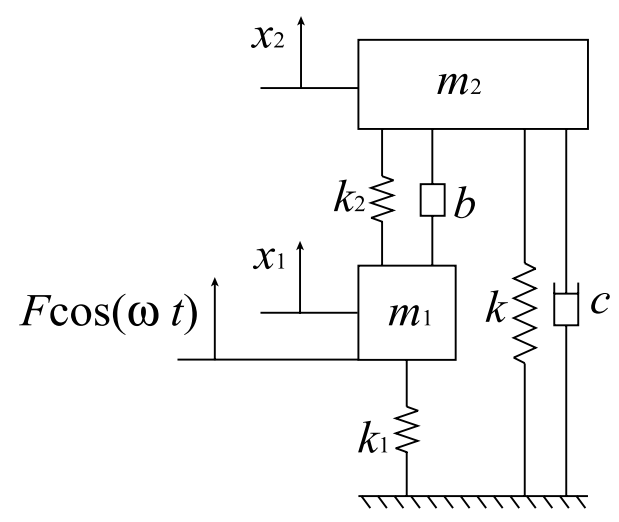




$$
\begin{gathered}
\omega_{1}=\sqrt{\frac{k_{1}}{m_{1}}}, \quad \omega_{2}=\sqrt{\frac{k_{2}}{m_{2}}}, \quad \xi=\frac{c}{2 m_{2} \omega_{2}} \\
\mu=\frac{m_{2}}{m_{1}}, \quad \alpha=\frac{k}{k_{2}}, \quad f=\frac{F}{m_{1}}, \quad h=\frac{b}{m_{2}}
\end{gathered}
$$

Eq. (1) becomes

$$
\begin{aligned}
& \ddot{x}_{1}+\left(\omega_{1}^{2}+\mu \omega_{2}^{2}\right) x_{1}-\mu \omega_{2}^{2} x_{2}+\mu h \ddot{x}_{1}-\mu h \ddot{x}_{2}=f \cos (\omega t) \\
& \ddot{x}_{2}+\left(\alpha \omega_{2}^{2}+\omega_{2}^{2}\right) x_{2}-\omega_{2}^{2} x_{1}+2 \omega_{2} \xi \dot{x}_{2}-h \ddot{x}_{1}+h \ddot{x}_{2}=0
\end{aligned}
$$

\subsection{The Analytical Solution}

Letting $f \cos (\omega t)$ in Eq. (2) be represented by $f e^{j \omega t}$, the steady state solutions take the forms as

$$
x_{1}=X_{1} e^{j \omega t}, \quad x_{2}=X_{2} e^{j \omega t}
$$

Substituting (3) into (2), one could obtain

$$
X_{1}=\frac{A_{2} f}{A_{1} A_{2}-B_{1} B_{2}}, \quad X_{2}=-\frac{B_{2} f}{A_{1} A_{2}-B_{1} B_{2}}
$$

where

$$
\begin{aligned}
& A_{1}=-\omega^{2}+\omega_{1}^{2}+\mu\left(\omega_{2}^{2}-h \omega^{2}\right) \\
& A_{2}=-(h+1) \omega^{2}+\omega_{2}^{2}(\alpha+1)+2 j \omega \omega_{2} \xi \\
& B_{1}=\mu\left(h \omega^{2}-\omega_{2}^{2}\right) \\
& B_{2}=h \omega^{2}-\omega_{2}^{2}
\end{aligned}
$$

Introducing the parameters

$$
\lambda=\frac{\omega}{\omega_{1}}, \quad v=\frac{\omega_{2}}{\omega_{1}}, \quad X_{s t}=\frac{F}{k_{1}}
$$

Based on the above analysis, the amplitude amplification factor of the primary system should be

$$
A=\left|\frac{X_{1}}{X_{s t}}\right|=\sqrt{\frac{A_{3}^{2}+B_{3}^{2} \xi^{2}}{C_{3}^{2}+D_{3}^{2} \xi^{2}}}
$$

where 
Fig. 2 The normalized amplitude-frequency curves

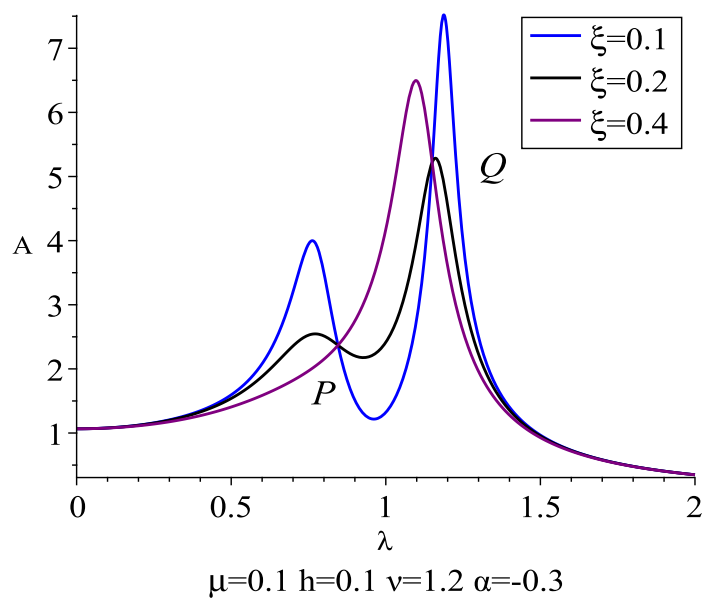

$$
\begin{aligned}
& A_{3}=\beta_{1}-\lambda^{2}(h+1), \quad B_{3}=2 \lambda \nu, \quad D_{3}=2 \lambda \nu\left(\beta_{2}-\lambda^{2} \beta_{3}\right) \\
& C_{3}=\left(\beta_{1}-\lambda^{2}(h+1)\right)\left(\beta_{2}-\lambda^{2} \beta_{3}\right)-\mu\left(h \lambda^{2}-v^{2}\right)^{2} \\
& \beta_{1}=v^{2}(\alpha+1), \quad \beta_{2}=\mu v^{2}+1, \quad \beta_{3}=\mu h+1
\end{aligned}
$$

\subsection{Parameters Optimization}

By simple deduction of (6), it can be found that the normalized amplitude-frequency curve under the different damping ratios as $0.1,0.2$ and 0.4 given in Fig. 2 will pass through two points which are independent of the damping ratio. And it could be clearly seen that there exist two commonly fixed points $P$ and $Q$ on all the curves, which are independent of the damping ratio. Thus, based on fixed point theory, the optimum natural frequency ratio can be obtained by adjusting the responses at $P$ and $Q$ to the same level. Then making $P$ and $Q$ as the maximum values of the amplitudefrequency curve to obtain the optimum damping ratio.

Due to the fixed-point theory, there exist two equal values if $\xi \rightarrow \infty$ and $\xi \rightarrow 0$ in Eq. (6)

$$
\left|\frac{A_{3}}{C_{3}}\right|=\left|\frac{B_{3}}{D_{3}}\right|
$$

i.e.

$$
\frac{\beta_{1}-\lambda^{2}(h+1)}{\left(\beta_{1}-\lambda^{2}(h+1)\right)\left(\beta_{2}-\lambda^{2} \beta_{3}\right)-\mu\left(h \lambda^{2}-v^{2}\right)^{2}}= \pm \frac{1}{\beta_{2}-\lambda^{2} \beta_{3}}
$$

It could be found that there is no meaning when the right part of (9) is positive. Accordingly, taking the negative one and simplifying the equation, one can get 


$$
\lambda^{4}+a_{1} \lambda^{2}+a_{2}=0
$$

where

$$
\begin{aligned}
& a_{1}=\frac{2\left(\mu \nu^{2} h-\beta_{1} \beta_{3}-\beta_{2}(h+1)\right)}{2(h+1) \beta_{3}-\mu h^{2}} \\
& a_{2}=\frac{\mu \nu^{4}-2 \beta_{1} \beta_{2}}{2(h+1) \beta_{3}-\mu h^{2}}
\end{aligned}
$$

Supposing the roots of Eq. (10) are $\lambda_{P}$ and $\lambda_{Q}$, one can get the following equation

$$
\lambda_{P}^{2}+\lambda_{Q}^{2}=-\frac{2\left(\mu \nu^{2} h-\beta_{1} \beta_{3}-\beta_{2}(h+1)\right)}{2(h+1) \beta_{3}-\mu h^{2}}
$$

In order to get the optimum natural frequency ratio, adjusting the values at $P$ and $Q$ to be the same

$$
\frac{1}{\beta_{2}-\beta_{3} \lambda_{P}^{2}}=-\frac{1}{\beta_{2}-\beta_{3} \lambda_{Q}^{2}}
$$

Simplifying Eq. (12) leads to

$$
\lambda_{P}^{2}+\lambda_{Q}^{2}=\frac{2 \beta_{2}}{\beta_{3}}
$$

Combining Eqs.(11) and (13), the optimum natural frequency ratio can be obtained

$$
v_{o p t}=\sqrt{\frac{\beta_{3}+h}{b_{1}}}
$$

where $b_{1}=\alpha \beta_{3}^{2}-\mu \beta_{3}+1$. For convenience, we denote

$$
b_{2}=\alpha \beta_{3}+1, \quad b_{3}=(h+2) \beta_{3}+h, \quad b_{4}=\beta_{3}(1-h \alpha)
$$

Then the two fixed points can be obtained

$$
\lambda_{P}^{2}=\frac{b_{2} b_{3}-b_{4} \sqrt{\mu b_{3}}}{b_{1} b_{3}}, \quad \lambda_{Q}^{2}=\frac{b_{2} b_{3}+b_{4} \sqrt{\mu b_{3}}}{b_{1} b_{3}}
$$

Based on the optimum natural frequency ratio, the amplitude amplification factor at $P$ and $Q$ can be obtained

$$
\left.A\right|_{\lambda_{P}, \lambda_{Q}}=\frac{b_{1} b_{3}}{\beta_{3} b_{4} \sqrt{\mu b_{3}}}
$$

Next, to make the maximum amplitude of the primary system at the fixed points, the damping ratio is adjusted. The condition can be achieved when the derivatives of the amplitude amplification factor are zero at the two fixed points 


$$
\frac{\partial A^{2}}{\partial \lambda_{P}^{2}}=0, \quad \frac{\partial A^{2}}{\partial \lambda_{Q}^{2}}=0
$$

Solving Eq. (17) and substituting the optimum natural frequency ratio into the results one can get

$$
\begin{aligned}
& \xi_{P}^{2}=\frac{\mu(1-\alpha h)^{2}\left(2 b_{3}-\beta_{3}-h+2 h \sqrt{\mu b_{3}}\right)}{4\left(b_{2} b_{3}-b_{4} \sqrt{\mu b_{3}}\right)} \\
& \xi_{Q}^{2}=\frac{\mu(1-\alpha h)^{2}\left(2 b_{3}-\beta_{3}-h-2 h \sqrt{\mu b_{3}}\right)}{4\left(b_{2} b_{3}+b_{4} \sqrt{\mu b_{3}}\right)}
\end{aligned}
$$

Taking an average of $\xi_{P}$ and $\xi_{Q}$, one can get the optimum damping ratio

$$
\xi_{\text {opt }}=\sqrt{\frac{\xi_{P}^{2}+\xi_{Q}^{2}}{2}}=\frac{1-\alpha h}{2} \sqrt{\frac{\mu b_{2}\left(2 b_{3}-\beta_{3}-h\right)+2 \mu^{2} h b_{4}}{b_{2}^{2} b_{3}-\mu b_{4}^{2}}}
$$

From the above analysis, we can see that this result cannot exactly make the points $P$ and $Q$ as the maximum values of the amplitude-frequency curve. But this approximation can be accepted as a simple design law. Considering Eqs. (14) and (19), there still exist adjustable parameters in the optimum natural frequency ratio and damping ratio. According to the characteristics of the negative stiffness system, it can be achieved when the negative stiffness material is applied by preload, which could be realized by pre-compressed member or inverted pendulum. The preload will cause a pre-displacement of the primary system, therefore, an approximation method is adopted to select the pre-displacement as the amplitude at the fixed point, which means the response to zero-frequency excitation is the same as the response at the fixed points.

$$
A=\left|\frac{X_{1}}{X_{s t}}\right|=\frac{b_{1} b_{3}}{\beta_{3} b_{4} \sqrt{\mu b_{3}}}=\frac{b_{1}(1+\alpha)}{b_{1}+\alpha \beta_{3}\left(\alpha \beta_{3}+1\right)}
$$

Solving Eq. (20), we can obtain

$$
\begin{aligned}
& \alpha_{1}=-\frac{\beta_{3}-\sqrt{\mu b_{3}}}{\beta_{3}}, \quad \alpha_{2}=-\frac{\beta_{3}+\sqrt{\mu b_{3}}}{\beta_{3}} \\
& \alpha_{3}=-\frac{\beta_{3}+1-\sqrt{\mu b_{3}}}{2 \beta_{3}}, \quad \alpha_{4}=-\frac{\beta_{3}+1+\sqrt{\mu b_{3}}}{2 \beta_{3}}
\end{aligned}
$$

Considering Eqs. (14) and (19), one can find that taking $\alpha_{2}$ and $\alpha_{4}$ as the negative stiffness ratio will make the optimum natural frequency ratio as imaginary number and taking $\alpha_{3}$ as the negative stiffness will make the optimum damping ratio infinity. So $\alpha_{1}$ is choosed as the optimum negative stiffness ratio, namely 


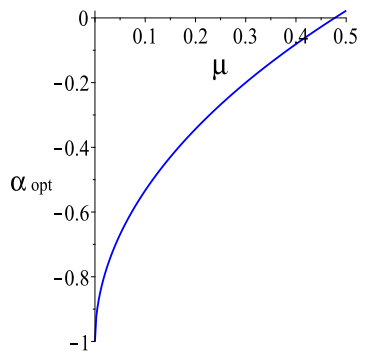

(a)

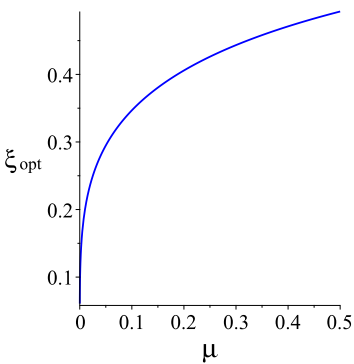

(b)

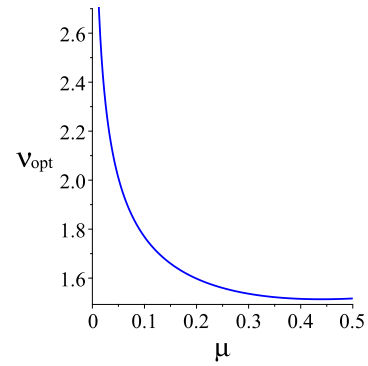

(c)

Fig. 3 The relationship between mass ratio and optimum parameters

$$
\alpha_{o p t}=-\frac{\beta_{3}-\sqrt{\mu b_{3}}}{\beta_{3}}
$$

Then according to the parameter optimization procedures, the three adjustable parameters of the DVA are analytically obtained.

Actually, in terms of practicality, we give the relationships between the optimum parameters in Eqs.(14), (19), and (22) with the mass ratio in Fig. 3. Based on the analysis of the figure, we can find that the negative stiffness ratio plays a small role in the consideration of the system stability.

\subsection{The Best Working Range of Inerter}

According to the calculation, when the inerter-mass ratio is not appropriate, the optimal solution will cause system instability, so it can be deduced that in the optimal design process of dynamic vibration absorber, under the premise of ensuring system stability, there is an optimal working range of inerter-mass ratio. The coefficients of existing inerter-mass ratio elements are all positive and the mass ratio is positive. Considering each optimal parameter and its calculation process, the working range of inerter-mass ratio should satisfy that the denominator of each expression is not equal to 0 , the part under the square root is greater than 0 , and the optimal frequency ratio $v_{\text {opt }}$ and optimal damping ratio $\xi_{\text {opt }}$ are greater than 0 .

Substituting negative stiffness ratio $\alpha_{\text {opt }}$ into optimal frequency ratio, yields

$$
v_{\text {opt }}=\sqrt{\frac{\beta_{3}+h}{\beta_{3} \sqrt{\mu b_{3}}-\mu\left(b_{3}-\beta_{3}\right)}}
$$

From above analysis, inerter-mass ratio $h$ should simultaneously satisfy

$$
\alpha_{\text {opt }}<0, \quad v_{\text {opt }}>0, \quad \xi_{\text {opt }}>0
$$

Solving equation (24), one can obtain 


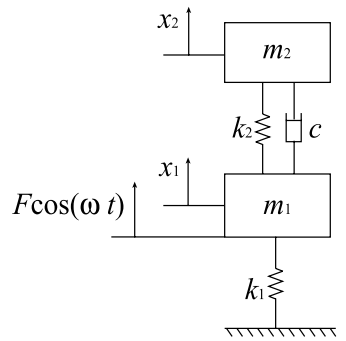

(a)

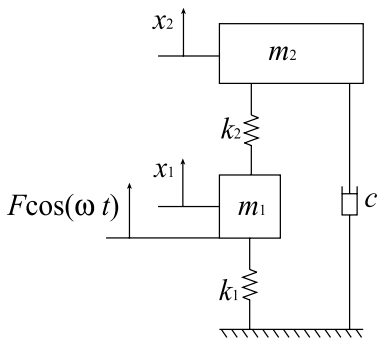

(b)

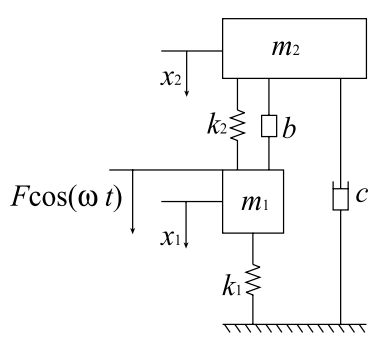

(c)

Fig. 4 Three typical models of DVAs: a The type by Den Hartog; $\mathbf{b}$ The type by Ren; $\mathbf{c}$ The type by Reference 14

Table 1 The formulas of the three typical models of DVAs

\begin{tabular}{lll}
\hline Model of DVA & $v_{\text {opt }}$ & $\xi_{\text {opt }}$ \\
\hline Den Hartog & $\frac{1}{1+\mu}$ & $\sqrt{\frac{3 \mu}{8(1+\mu)}}$ \\
Ren & $\sqrt{\frac{1}{1-\mu}}$ & $\sqrt{\frac{3 \mu}{8(1-0.5 \mu)}}$ \\
Reference 14 & $\sqrt{\frac{1+h+\mu h}{1-\mu-\mu^{2} h}}$ & $\sqrt{\frac{\mu(2 \mu(1+\mu h)+\mu)}{4\left(\mu(1+\mu h)+\mu-\mu^{2}(1+\mu h)\right)}}$ \\
\hline
\end{tabular}

$$
0<h<\frac{1-2 \mu}{2 \mu^{2}}
$$

\section{Comparisons of the Control Performances}

In order to verify the vibration reduction effect of the dynamic vibration absorber model proposed in this paper, a comparison was made between the classic Voigt dynamic vibration absorber, Ren-type dynamic vibration absorber and IR1 dynamic vibration absorber with inerter-mass element attached in reference [14]. The three comparative vibration absorber models are shown in Fig. 4, and the optimal parameter formula corresponding to each model is shown in Table $1[4,17]$.

\subsection{The Comparison with Other DVAs Under Sinusoidal Excitation}

Many equipment in engineering, especially rotating machinery, is often subjected to harmonic excitation, therefore the vibration reduction effect of each model under different excitation frequencies is firstly compared. The mass ratio is taken $\mu=0.1$ and $\mu=0.05$ for all models, and the inerter-mass ratio is taken $h=0.1$ for the model in reference [14] and this paper. Relevant parameters are calculated according to the optimal parameter design formula previously deduced and existing in Table 1, and the displacement response of the primary system under different frequency harmonic 


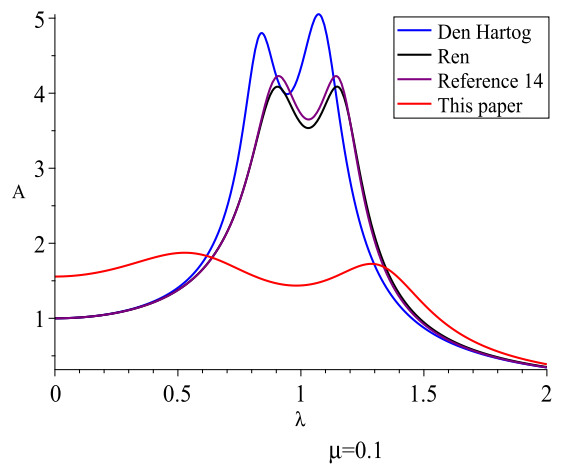

(a)

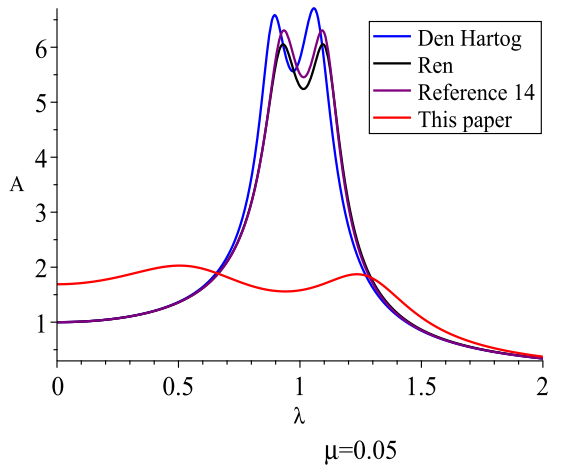

(b)

Fig. 5 The comparison with other DVAs

excitation is simulated. The normalized displacement amplitude-frequency curves of each model are obtained, as shown in Fig. 5.

It must be pointed out that we adopt approximation in the optimum damping ratio, thus the three amplitudes on zero-frequency excitation, fixed point $P$ and $Q$ are not exactly the same.

Through the above analysis, we can draw conclusions:

(1) According to the comparison, we can clearly find that the DVA presented in this paper can not only broaden the frequency range of vibration absorption, but also greatly reduce the amplitude of the primary system.

(2) By comparing with the traditional Den Hartog and Ren DVA, we can find that the negative grounding stiffness and inerter play a greater role in vibration absortion. Furthermore, compared with DVA in reference [14], it is found that the negative grounding stiffness is more beneficial to reduce the amplitude of the primary system.

(3) The presented DVA is not so sensitive to the mass ratio, which means one can get a better control performance using a smaller absorber mass.

\subsection{The Comparison with DVAs Under Random Excitation}

It is more important and meaningful to investigate the system response under random excitation, especially when DVA is applied in earthquake and civil

Fig. 6 A single degree system under random excitation

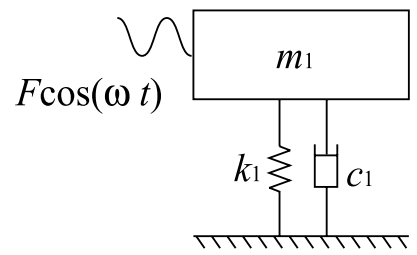


engineering. A further study is conducted on the DVA under random excitation in this subsection. When a single degree-of-freedom system (SDOF, the primary system) shown in Fig. 6 is subjected to random excitation with zero mean and power spectral density as $S(\omega)=S_{0}$, the mean square response of the SDOF system can be got as [3]

$$
\sigma^{2}=\frac{\pi S_{0}}{2 \omega_{1}^{3} \xi}
$$

Considering Eq. (26), one can find that the mean square response will become infinity if the damping coefficient approaches zero. Therefore, it is necessary to connect a DVA to reduce the system response.

Considering the primary system subjected to white noise excitation with zero mean value and constant power spectral density $S(\omega)=S_{0}$. Then, the power spectral density function of the displacement response of the above four models is as follows.

$$
\begin{aligned}
& S_{V}(\omega)=\left|X_{V_{1}}\right|^{2} S_{0}, \quad S_{R}(\omega)=\left|X_{R_{1}}\right|^{2} S_{0} \\
& S_{I R 1}(\omega)=\left|X_{I R 1_{1}}\right|^{2} S_{0}, \quad S_{N}(\omega)=\left|X_{N_{1}}\right|^{2} S_{0}
\end{aligned}
$$

where the subscript $V, R, I R 1$ and $N$ represent for the Den Hartog type DVA, the model by Ren, the model by reference [14] and the DVA in this paper respectively. According to the optimal parameters in literatures $[4,17]$, the mean square response of the primary system of these DVAs can be deduced as

$$
\begin{aligned}
\sigma_{V}^{2}=\int_{-\infty}^{+\infty} S_{V}(\omega) \mathrm{d} \omega & =S_{0} \int_{-\infty}^{+\infty}\left|X_{V_{1}}\right|^{2} \mathrm{~d} \omega=\frac{\pi S_{0} Y_{V}}{2 \omega_{1}^{3} \mu \xi \nu} \\
\sigma_{R}^{2}=\int_{-\infty}^{+\infty} S_{R}(\omega) \mathrm{d} \omega & =S_{0} \int_{-\infty}^{+\infty}\left|X_{R_{1}}\right|^{2} \mathrm{~d} \omega=\frac{\pi S_{0} Y_{R}}{2 \omega_{1}^{3} \mu \xi \nu^{5}} \\
\sigma_{I R 1}^{2}=\int_{-\infty}^{+\infty} S_{I R 1}(\omega) \mathrm{d} \omega & =S_{0} \int_{-\infty}^{+\infty}\left|X_{I R 1_{1}}\right|^{2} \mathrm{~d} \omega \\
& =\frac{\pi S_{0} Y_{I R 1}}{2 \omega_{1}^{3} \mu \xi \nu\left(v^{2}-h_{I R 1}\right)^{2}} \\
\sigma_{N}^{2}=\int_{-\infty}^{+\infty} S_{N}(\omega) \mathrm{d} \omega & =S_{0} \int_{-\infty}^{+\infty}\left|X_{N_{1}}\right|^{2} \mathrm{~d} \omega \\
& =\frac{\pi S_{0} Y_{N}}{2 \omega_{1}^{3} \mu \xi \nu\left(v^{2}-h\right)^{2}\left(\alpha \mu \nu^{2}+\alpha+1\right)}
\end{aligned}
$$

where 


$$
\begin{aligned}
Y_{V}= & v^{4}(1+\mu)^{2}+v^{2}\left(4 \xi^{2}(1+\mu)-\mu-2\right)+1 \\
Y_{R}= & v^{4}+v^{2}\left(4 \xi^{2}+\mu-2\right)+1 \\
Y_{I R 1}= & v^{4}+v^{2}\left(4 \xi^{2}\left(1+\mu h_{I R 1}\right)+\mu-2\left(1+h_{I R 1}\right)\right)+\left(1+h_{I R 1}\right)^{2} \\
Y_{N}= & v^{4} \gamma_{1}+v^{2} \gamma_{2}+(1+h)^{2}(\alpha+1) \\
\gamma_{1}= & (\mu h+1)^{2} \alpha^{3}+(2(1-\mu)(\mu h+1)+1) \alpha^{2} \\
& +\left(4 \mu \xi^{2}(\mu h+1)+(\mu-1)^{2}+2\right) \alpha+1 \\
\gamma_{2}= & -((\mu h+1)(h+2)+h) \alpha^{2}+\left(4 \xi^{2}(\mu h+1)-4(h+1)+2 \mu\right) \alpha \\
& +4 \xi^{2}(\mu h+1)-2(h+1)+\mu
\end{aligned}
$$

The mean square response of the primary system under the optimal parameters can be respectively obtained for $\mu=0.1, h=0.1, h_{I R 1}=0.1$ as follows

$$
\begin{aligned}
\sigma_{R}^{2} & =\frac{5.780 \pi S_{0}}{\omega_{1}^{3}}, & \sigma_{V}^{2} & =\frac{6.401 \pi S_{0}}{\omega_{1}^{3}} \\
\sigma_{I R 1}^{2} & =\frac{5.958 \pi S_{0}}{\omega_{1}^{3}}, & \sigma_{N}^{2} & =\frac{1.595 \pi S_{0}}{\omega_{1}^{3}}
\end{aligned}
$$

It is shown that the DVA with negative stiffness and inerter-mass in this paper has the minimum mean square response, which means the presented model can get a better performance than other DVAs even under the random excitation. Moreover, the presented model is still superior to other three DVAs if different mass ratios are selected.

Furthermore, we construct the $50 \mathrm{~s}$ random excitation, which is composed of 5000 normalized random numbers with zero mean and unit variance. The time history of random excitation is shown in Fig. 7. We take the primary mass $m_{1}=1 \mathrm{~kg}$, the stiffness $k_{1}=100 \mathrm{~N} / \mathrm{m}$, the mass of the vibration absorber is $m_{2}=0.1 \mathrm{~kg}$,

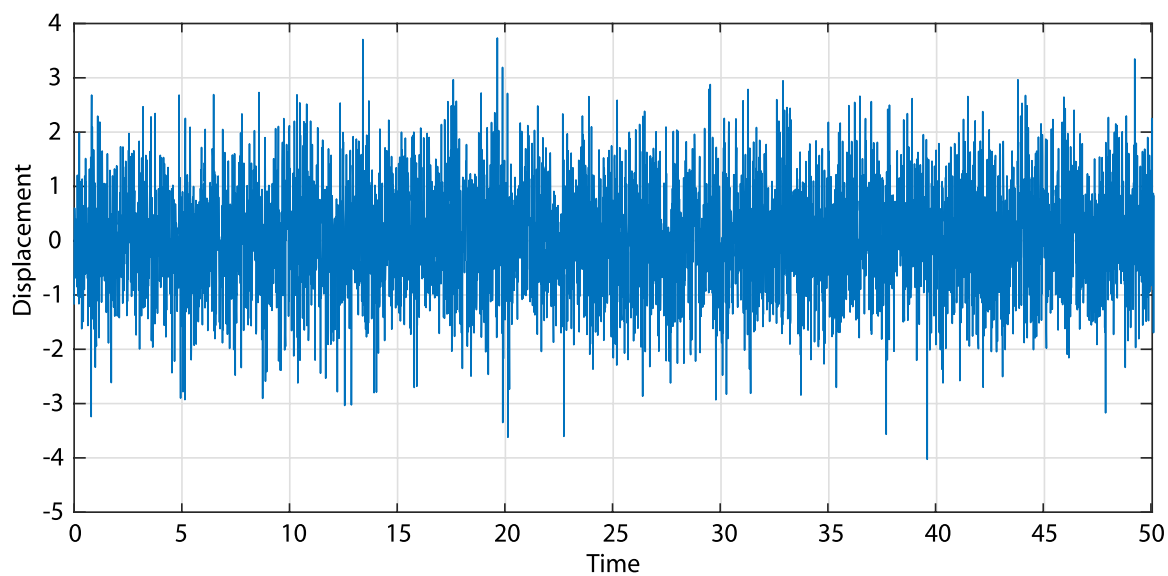

Fig. 7 The time history of the random excitation 


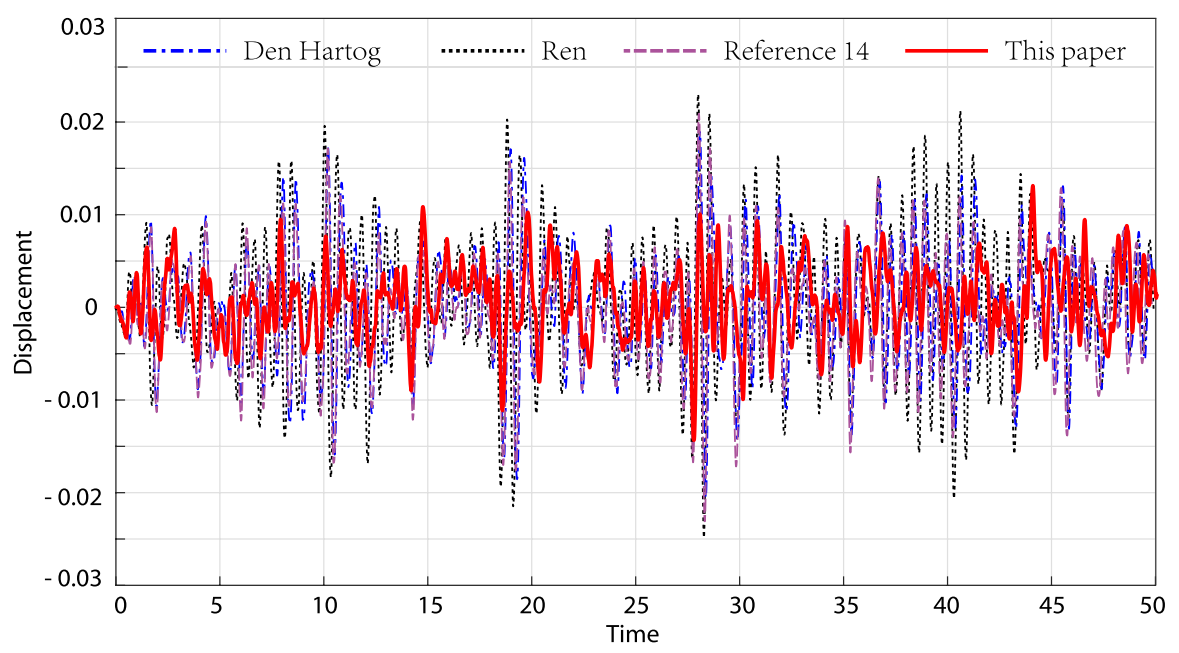

Fig. 8 Comparison of time domain dynamic responses for the primary system with different DVAs

according to Tab. 1 and the derivation process of this paper, the optimal parameters can be obtained. Based on the fourth-order Runge-Kutta method, the response of the primary systems without DVA and with different DVAs can be obtained. Figure 8 shows the displacement response of the four different dynamic vibration absorbers attached to the primary system.

From Fig. 8, it could be concluded that DVA in this paper could present better control performance than other DVAs, even when the primary system is subjected to random excitation. Comparing with the traditional Den Hartog and Ren DVA, we can find that the negative grounding stiffness and inerter play a greater role in vibration absortion. Furthermore, when the DVA is attached with the negative grounding stiffness, compared with DVA in reference [14], it is found that the DVA presented in this paper has better control performance under random excitation.

\section{Conclusions}

In this paper, a novel dynamic vibration absorber model with inerter-mass and grounding negative stiffness is proposed. Based on the fixed point theory, the optimal frequency ratio, approximate optimal damping ratio and optimal negative stiffness ratio are obtained. It is found that when inerter-mass and negative grounding stiffness act together, the system will be unstable when the improper inerter-mass coefficient is used in the optimal design process, thus the optimum working range of inerter-mass is obtained, which provides reference for the design of new vibration absorber in practical production. Compared with other types of dynamic vibration absorbers, the proposed DVA can greatly reduce the resonance amplitude and broaden the vibration frequency range. 
Open Access This article is distributed under the terms of the Creative Commons Attribution 4.0 International License (http://creativecommons.org/licenses/by/4.0/), which permits unrestricted use, distribution, and reproduction in any medium, provided you give appropriate credit to the original author(s) and the source, provide a link to the Creative Commons license, and indicate if changes were made.

Author Contributions All authors typed, read, reviewed, and approved the final manuscript.

Funding The research project is supported by National Natural Science Foundation of China (11772007, 11290152) and also supported by Beijing Natural Science Foundation (1172002, Z180005).

Availability of data and materials No data were used for this work.

\section{Declarations}

Conflict of interest The authors declare no conflict of interest.

Open Access This article is licensed under a Creative Commons Attribution 4.0 International License, which permits use, sharing, adaptation, distribution and reproduction in any medium or format, as long as you give appropriate credit to the original author(s) and the source, provide a link to the Creative Commons licence, and indicate if changes were made. The images or other third party material in this article are included in the article's Creative Commons licence, unless indicated otherwise in a credit line to the material. If material is not included in the article's Creative Commons licence and your intended use is not permitted by statutory regulation or exceeds the permitted use, you will need to obtain permission directly from the copyright holder. To view a copy of this licence, visit http://creativecommons.org/licen ses/by/4.0/.

\section{References}

1. Frahm,H.: Device for damping vibrations of bodies, U.S. Patent 089958, 10-30(1909)

2. Ormondroyd, J., Den Hartog, J.P.: The theory of the dynamic vibration absorber. J. Appl. Mech. 50, 9-22 (1928)

3. Den Hartog, J.P.: Mechanical Vibrations, pp. 112-132. McGraw-Hall Book Company, New York (1947)

4. Ren, M.Z.: A variant design of the dynamic vibration absorber. J. Sound Vib. 245, 762-770 (2001)

5. Asami, T.: Closed-form exact solution tooptimization of dynamic vibration absorbers: Application to different transfer functions and damping systems. J. Vib. Acoust. 125, 398-405 (2003)

6. Nishihara, O., Asami, T.: Close-form solutions to the exct optimizations of dynamic vibration absorber(minimizations of the maximum amplitude magnification factors). ASME J. Vib. Acoust. 124, 576-582 (2002)

7. Asami, T., Nishihara, O., Baz, A.M.: Analytical solutionstoand H2 optimization of dynamic vibration absorbers attached to damped linear systems. J. Vib. Acoust. 124, 284-295 (2002)

8. Acar, M.A., Yilmaz, C.: Design of an adaptive-passive dynamic vibration absorber composed of a string-mass system equipped with negative stiffness tension adjusting mechanism. J. Sound Vib. 332, 231-245 (2013)

9. Yang, J., Xiong, Y.P., Xing, J.T.: Dynamics and power flow behaviour of a nonlinear vibration isolation system with a negative stiffness mechanism. J. Sound Vib. 332, 167-183 (2013)

10. Shen, Y.J., Peng, H.B., Li, X.H., Yang, S.P.: Analytically optimal parameters of dynamic vibration absorber with negative stiffness. Mech. Syst. Signal Process. 85, 192-203 (2017)

11. Shen, Y.J., Xing, Z.Y., Yang, S.P., Sun, J.Q.: Parameters optimization for a novel dynamic vibration absorber. Mech. Syst. Signal Process. 133, 106282 (2019)

12. Zhou, S.Y., Jean-Mistral, C., Chesne, S.: Closed-form solutions to optimal parameters of dynamic vibration absorbers with negative stiffness under harmonic and transient excitation. Int. J. Mech. Sci. 157-158, 528-541 (2019) 
13. Wang, X.R., He, T., Shen, Y.J., Shan, Y.C., Liu, X.D.: Parameters optimization and performance evaluation for the novel inerter-based dynamic vibration absorbers with negative stiffness. J. Sound Vib. 463, 114941 (2019)

14. Wang, X.R., Liu, X.D., Shan, Y.C., Shen, Y.J., He, T.: Analysis and optimization of the novel inerter-based dynamic vibration absorbers. IEEE Access 6, 2844086 (2018)

15. Chen, M.Z.Q., Hu, Y.L., Huang, L.X., Chen, G.R.: Influence of inerter on natural frequencies of vibration systems. J. Sound Vib. 333, 1874-1887 (2014)

16. Hu, Y.L., Chen, M.Z.Q., Shu, Z., Huang, L.X.: Analysis and optimisation for inerter-based isolators via fixed-point theory and algebraic solution. J. Sound Vib. 346, 17-36 (2015)

17. Gioacchino, A., Giuseppe, F.: Improved inerter-based vibration absorbers. Int. J. Mech. Sci. 192, 106087 (2021)

18. Brzeski, P., Kapitaniak, T., Perlikowski, P.: Novel type of tuned mass damper with inerter which enables changes of inertance. J. Sound Vib. 349, 56-66 (2015)

19. Willian, M.K., Paulo, J.P.G., Diego, F.L.R., et al.: Inerter-like devices used for vibration isolation: a historical perspective. J. Franklin Inst. 358, 1070-1086 (2021)

20. De Domenico, D., Ricciardi, G.: An enhanced base isolation system equipped with optimal tuned mass damper inerter (TMDI). Earthq. Eng. Struct. Dynam. 47, 1169-1192 (2018)

21. Kim,S. Y.: Lee,C. H.: Analysis and optimization of multiple tuned mass dampers with coulomb dry friction, Eng. Struct., 209 (2019)

22. Sun, X.T., Jing, X.J.: Analysis and design of a nonlinear stiffness and damping system with a scissor-like structure. Mech. Syst. Signal Process. 66-67, 723-742 (2016)

23. Bian, J., Jing, X.J.: A nonlinear X-shaped structure based tuned mass damper with multi-variable optimization (X-absorber). Commun. Nonlinear Sci. Numer. Simul. 99, 105829 (2021) 